\title{
Effects of drought stress on relative water, chlorophyll and proline content in tolerant and susceptible genotypes of lentil (Lens culinaris Medik.)
}

\author{
A. P. DASH, ${ }^{1}$ D. K. DE, R. NATH, ${ }^{2}$ A. SARKAR, S. MOHANTY AND \\ ${ }^{1}$ P. K. BHATTACHARYYA \\ Department of Agronomy, ${ }^{1}$ Department of Genetics \& Plant Breeding, Faculty of Agriculture, \\ Bidhan Chandra Krishi Viswavidyalaya, Mohanpur-741252, India \\ ${ }^{2}$ Food Legume Breeder, ICARDA
}

Received : 15.11.2019 ; Revised :13.04.2020 ; Accepted : 10.05.2020

DOI: https://doi.org/10.22271/09746315.2020.v16.i1.1293

\begin{abstract}
The present experiment was carried out in the Department of Genetics and Plant Breeding, Bidhan Chandra Krishi Viswavidyalaya, West Bengal, India to study the effect of imposed drought on relative water content, chlorophyll content and proline accumulation in five drought tolerant (PL-406, IPL-324, LL-1146, IPL-325, K-75) and five drought sensitive (L111220, LP-112, ILL-10803, KLS-113, KLS-107) genotypes of lentil (Lens culinaris Medik.). For this purpose, the genotypes were grown under both control (0.0 bar) and stress under drought (-9 bar) condition by using PEG- 6000 as osmoticum. The experiment was laid out in an asymmetrical factorial completely randomized design with three replications. Roots, shoots and leaves of ten days old seedlings were harvested and physiological \& biochemical analysis were carried out for the above parameters. From the study, it was revealed that relative water content (RWC) and chlorophyll content (chl a, Chl b \& total Chl) were reduced, while proline content was increased due to imposition of drought stress with compared to respective control. However, the tolerant genotypes exhibited lower relative reduction for RWC and chlorophyll content and proline content increased more as compared to susceptible ones under drought stress condition. The results suggested that higher levels of RWC, chlorophyll content and proline accumulation in tolerant variety of lentil could play an important role in drought stress tolerance.
\end{abstract}

Keywords : Chlorophyll, drought, lentil, proline, RWC

Climate change is a global phenomenon that renders adverse impact on agriculture. In Agriculture, drought is one of the most devastating abiotic stresses (Vinocur and Altman, 2005). During domestication of crop plants and their improvement, much emphasis was given for selection on different desirable characters including yield and tolerance to drought was unlikely to be one of such criteria for selection. The global water scarcity and increasing demand for non-agricultural uses of water, area expansion under irrigation does not appear to be a realistic scenario to address the challenge of food security in the developing countries. Thus, the scenario as a whole reveals that when the global food requirement is increasing rapidly, the water stress is also increasing alarmingly. Food production, therefore, in the twentyfirst century will have to rely more on the release of varieties with higher potentiality to combat drought and with higher yield stability (Borlaug, 2007). For this, it is inevitable to understand the functioning capacity of drought tolerant plants under drought stress condition. Different parameters like RWC, proline, chlorophyll content have been considered as markers of such. Leaf chlorophyll content found to be positively correlated with soil moisture content, while negative correlations were obtained with proline and anthocyanin content (Baroowa and Gogoi, 2013). Also, in Chickpea relative the Relative water content and Chlorophyll a and b content decreased whereas Proline content increased drastically under drought stress condition (Gokmen and Ceyhan, 2018).

Lentil generally possesses an inbuilt mechanism to tolerate drought but there are known degrees of differences between varieties. During the course of evolution, several mechanisms has been developed by plants that help in perceiving the stresses and their physiological and metabolic activities regulated to combat them. An understanding of plants' physiological and biochemical mechanism to combat water stress can aid in identifying cultivars that can profitably be used to produce sustainably high yield. However, studies on different physiological and biochemical factors during stress under drought in a drought tolerant crop like lentil still remain elusive. Therefore, the present study was taken up to compare the changing status of chlorophyll, proline and relative water content in the seedlings of drought tolerant and sensitive genotypes due to imposition of PEG-6000 induced drought stress.

\section{MATERIALS AND METHODS}

\section{Seed material}

Seeds of forty-eight genotypes of lentil (Lens culinaris Medik.) procured from AICRP on MULLaRP, Kalyani centre, Bidhan Chandra Krishi Viswavidyalaya 
(BCKV), Mohanpur, West Bengal, India were screened for drought tolerance during the period from first week of November to last week of January in two consecutive years (2013-14 and 2014-15) in the laboratory of the Department of Genetics and Plant Breeding, BCKV using PEG-6000 as osmoticum. Based on the performance of forty-eight genotypes in the laboratory with respect to different morphological characters at seedling stage and their respective drought tolerance efficiency, five most tolerant genotypes viz., PL-406, IPL-324, LL-1146, IPL-325 and K-75 and five most susceptible genotypes viz., L1112-20, LP-112, ILL10803, KLS-113 and KLS-107 were identified for their study on physiological and biochemical status to drought stress.

\section{Drought stress treatment}

Seventy two healthy, viable seeds of each lentil genotype were surface sterilized by immersing them in $70 \%$ ethanol for 2 minutes followed by thorough washing with distilled water. Twelve such seeds of a genotype were arranged in a row with even space over a glass plate $(20 \times 30 \mathrm{~cm})$ wrapped with a blotting paper. To prevent the seeds from sliding down when the set was kept in a slanted position in a stand, another glass strip ( $20 \times 2 \mathrm{~cm}$ ) was placed over the seeds with the help of a piece of thermocol at the two ends and guarder in such a way that the seeds remained in their position and the seedlings grew without any hindrance. The whole set was then placed in a transparent polythene bag. There were six such sets for each genotype; three sets represented three replications for control and the remaining as three replications for treatment. In the treatment plates, PEG solution of -9 bar water potential was used as germinating medium, while in the control plates pure distilled water was used for the purpose. The seeds were then allowed to germinate and grow for 10 days under indoor laboratory condition under sufficient light of 3000 lux, 70-80 \% relative humidity (RH) and at a temperature range of $20-25^{\circ} \mathrm{C}$. Six competitive seedlings were randomly selected from each plate and further analysis was carried out through destructive sampling for relative water content, chlorophyll content and proline content following Barr and Weatherley (1962), Arnon (1949) and Bates et al. (1973) respectively.

\section{RESULTS AND DISCUSSION}

\section{Relative water content (RWC)}

The data on relative water content in different plant parts of ten days old seedling of the above mentioned susceptible and tolerant genotypes grown under control ( 0.0 bar) and water stress (-9.0 bar) conditions have been presented in table 1 . All genotypes exhibited reduced relative water content in root due to imposition of water stress but such reduction was comparatively more in susceptible genotypes than in tolerant ones. Under control condition, only one genotype from tolerant group (LL-1146) and two genotypes (KLS-113 \& (L-1112-20) from susceptible group exhibited significantly higher mean RWC while under drought stress all the genotypes except PL-403 from tolerant group and none from the susceptible group exhibited significantly higher mean as revealed by CD (critical difference) value. The highest reduction with compared to respective control was observed in a tolerant genotype, K-75 (29.257 \%) followed by susceptible genotype, KLS- 107 (29.196 $\%)$ and the lowest was in a tolerant one, IPL-324 (11.054\%) followed by another tolerant genotype IPL325 (12.738 \%).

As observed in root in case of shoot also all the ten genotypes witnessed a reduction of RWC due to imposition of drought and it was much pronounced in susceptible genotypes. However, under control condition, two genotypes from tolerant group (PL-406 \& LL-1146) and only one genotype (KLS-113) from susceptible group exhibited significantly higher mean RWC but under water stress condition all the tolerant genotypes exhibited significantly higher mean but none from the susceptible group could do so. Interestingly, root was found to be more influenced by imposed drought than shoot.

In case of leaf, two genotypes from tolerant group (LL-1146 and K-75) and three (KLS-107, KLS-113 and L1112-20) from susceptible group under control, while three genotypes (IPL-324, LL-1146 and K-75) from control group and only one (LP-112) from susceptible group exhibited significantly higher mean as revealed by CD value. Though in all genotypes leaf RWC decreased, it was more in case of susceptible genotypes as compared to tolerant ones. Among the three characters the leaves were moderately affected as revealed by the relative reduction percentage.

Therefore, it may be concluded that water stress imposition reduced the relative water content of all the morphological parts of the seedlings (root, shoot, leaf), which has been reported earlier by Merah (2001) in case of wheat. The higher relative reduction of RWC in sensitive genotypes of lentil suggests more profound effect of drought stress on them as compared to tolerant ones. The possible cause of this reduction may be the loss of turgidity of cells under water stress. High relative water content is associated with the resistant mechanism to drought and more osmotic regulation or less elasticity of tissue cell wall results high relative water content (Ritchie et al., 1990)

Similar mechanism might have played role in the 
Table 1: Relative water content in different parts of lentil seedlings among tolerant and susceptible genotypes under control and drought condition.

\begin{tabular}{|c|c|c|c|c|c|c|c|c|c|}
\hline Character & & Root & & & Shoot & & & Leaf & \\
\hline Genotype & Control & PEG Solı & n RR (\%) & Control & PEG Soln & RR (\%) & Control & PEG Soln & RR (\%) \\
\hline PL-406 (T) & 81.784 & 63.271 & 21.645 & 84.392 & 76.015 & 9.926 & 82.357 & 67.777 & 17.703 \\
\hline IPL-324 (T) & 83.452 & 65.389 & 11.054 & 81.380 & 72.985 & 10.316 & 83.253 & 71.212 & 14.463 \\
\hline LL-1146 (T) & 85.474 & 76.026 & 20.062 & 86.471 & 80.221 & 7.228 & 84.141 & 75.420 & 10.365 \\
\hline IPL-325 (T) & 83.493 & 66.743 & 12.738 & 81.949 & 73.052 & 10.857 & 81.077 & 69.108 & 14.763 \\
\hline K-75 (T) & 83.170 & 72.576 & 29.257 & 80.130 & 73.137 & 8.727 & 83.697 & 73.600 & 12.064 \\
\hline KLS-107 (S) & 83.467 & 59.047 & 29.196 & 81.730 & 71.044 & 13.075 & 84.097 & 67.811 & 19.366 \\
\hline KLS-113 (S) & 85.657 & 60.649 & 23.113 & 83.253 & 72.014 & 13.500 & 84.477 & 66.940 & 20.759 \\
\hline ILL-10803 (S) & 83.164 & 63.942 & 24.259 & 80.156 & 68.073 & 15.074 & 83.220 & 64.775 & 22.164 \\
\hline LP-112 (S) & 80.173 & 60.724 & 27.111 & 78.920 & 70.080 & 11.201 & 80.310 & 70.060 & 12.763 \\
\hline L1112-20 (S) & 84.091 & 61.293 & 21.645 & 81.126 & 68.715 & 15.298 & 85.842 & 65.335 & 23.889 \\
\hline Mean B & 83.393 & 64.966 & 74.179 & 81.951 & 72.534 & 77.242 & 83.247 & 69.204 & 76.226 \\
\hline Factors & $\mathrm{SE}(\mathrm{m})$ & SE(d) & $\operatorname{LSD}(0.05)$ & SE(m) & SE(d) I & LSD(0.05) & SE(m) & SE(d) & LSD(0.05) \\
\hline Genotype (G) & 0.035 & 0.049 & 0.103 & 0.035 & 0.049 & 0.103 & 0.042 & 0.059 & 0.125 \\
\hline Treatment (T) & 0.015 & 0.022 & 0.046 & 0.015 & 0.022 & 0.046 & 0.019 & 0.027 & 0.056 \\
\hline G X T & 0.049 & 0.069 & 0.145 & 0.049 & 0.069 & 0.145 & 0.059 & 0.084 & 0.177 \\
\hline
\end{tabular}

Note:T= Morphologically tolerant $S=$ Morphologically susceptible $R R=$ Relative reduction

tolerant genotypes expressing significantly higher RWC for all the parts under drought stress in lentil. Earlier Bhardwaj and Yadav (2012) found significantly higher RWC in the tolerant variety under drought stress.

\section{Chlorophyll content}

The results obtained with respect to the content of chlorophyll 'a', chlorophyll 'b' and total chlorophyll per unit quantity of fresh leaf tissue from 10 days old seedling of the above mentioned genotypes grown under control ( 0.0 bar) and water stress ( -9.0 bar) condition presented in table 2 reveal significant inter-genotypic differences as indicated by the CD value. Such differential response might have arisen due to differential genetic makeup of different genotypes. The range of chlorophyll 'a' content in the seedlings grown under control in the susceptible group from $0.268 \mathrm{mg} \mathrm{g}^{-1}$ tissue to $0.464 \mathrm{mg} \mathrm{g}^{-1}$ tissue and between $0.280 \mathrm{mg} \mathrm{g}^{-1}$ tissue and $0.425 \mathrm{mg} \mathrm{g}^{-1}$ tissue in case of tolerant ones indicated no remarkable difference in content of chlorophyll 'a' in the two groups of genotypes. But when the seedlings were subjected to water stress, there was differential behavior in the genotypes belonging to the two groups. Both tolerant as well as susceptible genotypes expressed a reduction in chlorophyll 'a' content due to treatment. Considering the mean values it was observed that in case of control, two genotypes from tolerant group viz., PL-406 and IPL325 and three genotypes from susceptible group viz., KLS-113, LP-112 and L1112-20 exhibited significantly higher mean. While in case of treatment though the same two genotypes from tolerant group exhibited significantly higher mean, but two out of three genotypes (KLS-113, LP-112) from susceptible group could do so. However, the extent of reduction of chlorophyll 'a' content due to drought was higher in susceptible genotypes than in the tolerant ones. While the highest and the lowest reduction in the content of chlorophyll ' $a$ ' among the susceptible genotypes were from KLS-107 (37.771 \%) and LP-112 (16.164\%), the same from tolerant genotypes were from PL-406 (14.588\%) and K-75 (8.966 \%) respectively. Therefore, the tolerant genotypes revealed to be less affected due to imposition of drought with compared to the susceptible ones. Similar results in Soybean (Hossaina et al., 2014) and Faba bean (Siddiqui et al., 2015) have been reported earlier.

In case of chlorophyll ' $b$ ' the mean value under control varied between $0.145 \mathrm{mg} \mathrm{g}^{-1}$ and $0.342 \mathrm{mg} \mathrm{g}^{-1}$ in the susceptible group and between $0.132 \mathrm{mg} \mathrm{g}^{-1}$ and $0.250 \mathrm{mg} \mathrm{g}^{-1}$ in case of tolerant ones. The genotypes that expressed significantly higher mean for chlorophyll ' $a$ ' under control as well as under water stress condition of both the groups could do so for chlorophyll 'b' as well. As observed in case of chlorophyll ' $a$ ', both tolerant and susceptible genotypes expressed a reduction in chlorophyll 'b' content due to treatment and the extent of such reduction due to drought stress was higher in susceptible genotypes than in the tolerant ones. Highest reduction of chlorophyll ' $b$ ' content was observed in a susceptible genotype, ILL-10803 (43.448 \%), while lowest was in a tolerant genotype, IPL-325 (22.321\%). Such decrease in chlorophyll content under stresses due to drought may be considered a typical symptom of 
Dash et al.

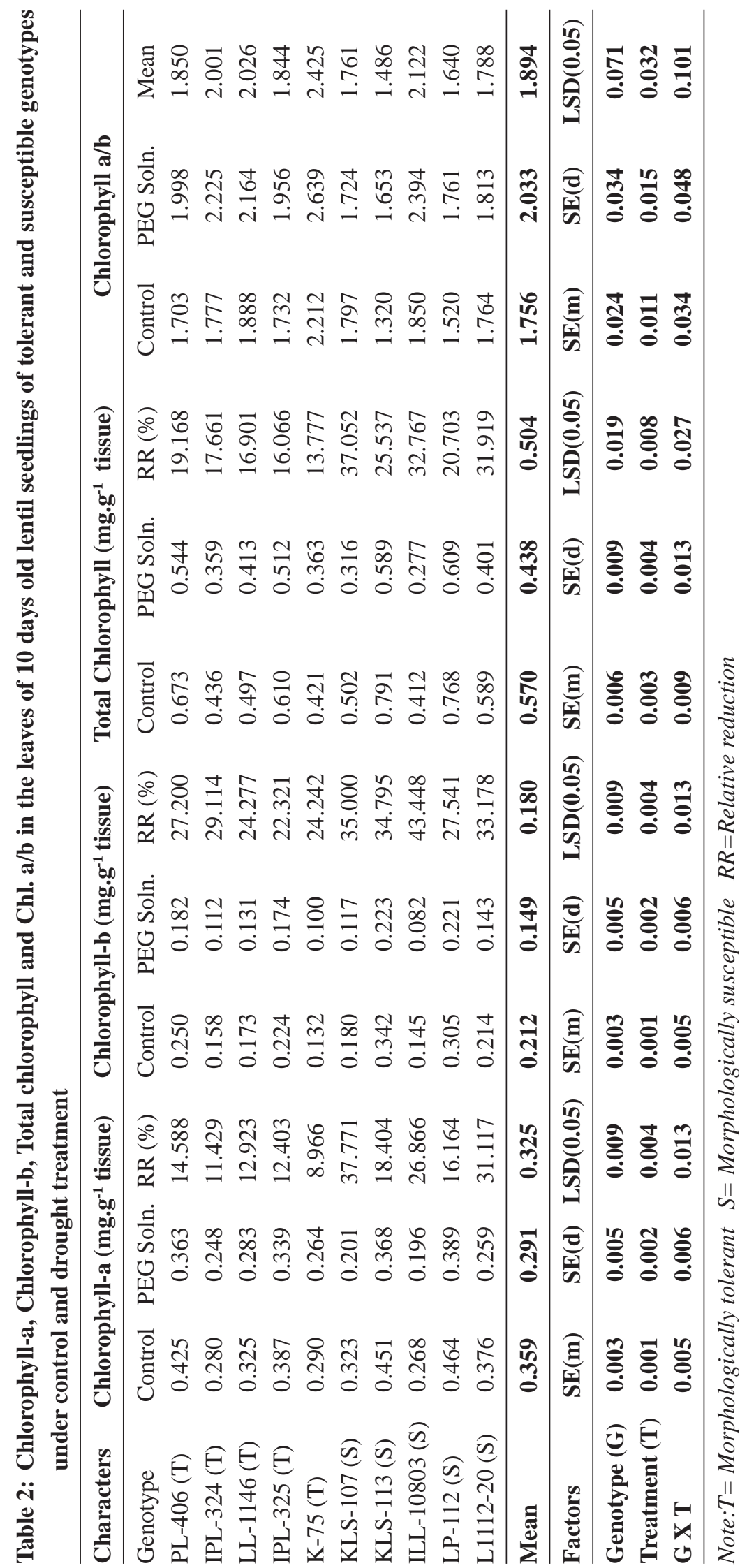


Table 3: Proline content in lentil seedlings of tolerant and susceptible genotypes under control and drought conditions

\begin{tabular}{|c|c|c|c|}
\hline & \multicolumn{2}{|c|}{ Proline content (micromole/g of fresh tissue) } & ) Mean \\
\hline Genotypes & Control & PEG soln. & \\
\hline PL-406 (T) & 110.520 & 249.070 & 179.795 \\
\hline IPL-324 (T) & 97.970 & 237.930 & 167.950 \\
\hline LL-1146 (T) & 166.100 & 361.810 & 263.955 \\
\hline IPL-325 (T) & 154.170 & 370.440 & 262.305 \\
\hline $\mathrm{K}-75(\mathrm{~T})$ & 113.540 & 320.660 & 217.100 \\
\hline KLS-107 (S) & 130.140 & 203.310 & 166.725 \\
\hline KLS-113 (S) & 108.320 & 211.320 & 159.820 \\
\hline ILL-10803 (S) & 115.220 & 190.860 & 153.040 \\
\hline LP-112 (S) & 80.170 & 139.760 & 109.965 \\
\hline L1112-20 (S) & 73.120 & 120.520 & 96.820 \\
\hline Mean & 114.927 & 240.568 & 177.748 \\
\hline Factors & $\mathrm{SE}(\mathrm{m})$ & $\mathrm{SE}(\mathrm{d})$ & $\operatorname{LSD}(0.05)$ \\
\hline Genotype(G) & 0.190 & 0.268 & 0.564 \\
\hline Treatment(T) & 0.085 & 0.120 & 0.252 \\
\hline G X T & 0.268 & 0.380 & 0.798 \\
\hline
\end{tabular}

Note: $T=$ Morphologically tolerant $S=$ Morphologically susceptible $R R=$ Relative reduction

oxidative stress and the result of pigment photo-oxidation and chlorophyll degradation.

Generally, the total chlorophyll content reflects the additive result of the two types of pigments studied, therefore, as expected, similar result was also observed for total chlorophyll content, where the susceptible genotypes expressed a higher relative reduction compared to tolerant ones. It may, therefore, be stated that chlorophyll 'a' and 'b' as well as total chlorophyll content reduced due to imposition of drought condition. However, in both the groups, the content of chlorophyll ' $b$ ' was more affected due to treatment than that of chlorophyll ' $a$ '. The present findings were in conformity with Kumar et al. (2011) who reported that drought stress reduced concentration of chlorophyll ' $b$ ' more than chlorophyll 'a'. Due to the imposition of water stress the ratio of two types of pigments (chlorophyll-a/b) was increased in all genotypes except KLS-107 indicating a higher reduction of chlorophyll 'b' as compared to chlorophyll 'a'. Such increase was observed to be more in tolerant genotypes than in susceptible ones.

According to Farooq et al. (2009) both the chlorophyll 'a' and 'b' are prone to soil dehydration. Reduction in total chlorophyll content was also reported by Aniat-ul-Haq et al. (2012); Al-Quraan et al. (2014) in lentil and Saglam et al. (2014) in chickpea. Manivannan et al. (2007) has reported a large decline in chlorophyll 'a', chlorophyll 'b' and the total chlorophyll content in different sunflower varieties due to water stress. According to Smirnoff (1995) reduction in chlorophyll under drought stress is mainly the result of damage to chloroplasts caused by reactive oxygen species. Therefore, in the present experiment imposition of drought might have caused damage to chloroplasts through reactive oxygen species.

\section{Proline content}

Many plant species accumulates proline as an adaptive response to adverse conditions under stress. Although, some researchers has raised the question for direct relationship between proline accumulation and stress adaptation, it is well accepted that proline content following stress is beneficial for the plant cell (Mattioli et al., 2009).

In the present experiment, analysis of proline content revealed a significant inter-genotypic difference in the seedlings grown under control as well as under water stress condition (Table 3). Among the susceptible genotypes grown under control, only KLS-107 recorded significantly higher mean, whereas two genotypes viz., LL-1146 and IPL-325 from the tolerant group exhibited significantly higher mean proline content. It was observed that the proline content increased in all the genotypes due to treatment but such increase was significant in four of the five tolerant genotypes viz., PL-406, LL-1146, IPL-325 and K-75 but none among the susceptible genotypes. Earlier Talukdar (2013) and Muscolo et al. (2014) in lentil and Rizvi et al. (2014) in chickpea reported proline accumulation under drought stress. Highest increase (182.42 \%) in proline accumulation observed in K-75 is a tolerant genotype and the lowest increase (56.224 \%) was in KLS-107, a susceptible genotype. Interestingly, K-75 and IPL-325 
revealed very low relative reduction for RWC and chlorophyll content also which indicated their consistency in tolerance to drought.

According to Kavi-Kishor et al. (2005), proline, the most important organic solutes in plants, maintains water content under stress condition as an osmoprotectant for membrane stabilization. In plants, the increased level of proline in PEG induced water stress may be an adaptation to overcome the stress. Generally, the plant tolerate stress by accumulating proline under stress condition to supply energy for growth and survival. (Sankar et al., 2007; Aktas et al., 2007).

Kumar et al. (2011) also found that during water stress the free proline content is increased and opined that proline is one of the common compatible osmolytes under water stress condition that helps to combat drought.

Therefore, from the present findings it may be concluded that all genotypes have potential to withstand drought through production of higher amount of proline under stress. But generally the tolerant genotypes have higher potential to withstand drought due to more accumulation of the amino acid. For further study on the inheritance pattern of drought tolerance, the genotypes like K-75, IPL-324, and IPL-325 from tolerant group and KLS-113 and LP-112 from susceptible group may be used in future.

In the present study it was observed that both relative water content and chlorophyll content reduced, while proline content increased in both susceptible and tolerant genotypes due to imposition of water stress. Though the difference in the three parameters under control condition was not remarkable among the genotypes within the two groups separately, a conspicuously higher value was observed for all parameters in tolerant genotypes compared to susceptible genotypes under water stress condition could be noticed.

\section{REFERENCES}

Aktas, L. Y., Akca, B. T. H. and Parlak, S. 2007. Role of Abscisic acid and Proline treatment on induction of antioxidant enzyme activities and drought tolerance responses of Laurus nobilis $L$. seedlings. Fen Bilimleri Dergisi, 28: 14-27.

Al-Quraan, N. A., Al-Sharbati, M., Dababneh, Y. and Al-Olabi, M. 2014. Effect of temperature, salt and osmotic stresses on seed germination and chlorophyll contents in lentil (Lens culinaris Medik.). Acta Hort., 10(54): 47-54.

Aniat-ul-Haq; Vamil, R. and Agnihotri, R. K. 2012. Effect of moisture stress (PEG) on biomass, total chlorophyll and proline content of lentil (Lens culinaris Medik.). Agric. Biol. Res., 28(1): 5663.
Arnon, D. I. 1949. Copper enzymes in isolated chloroplasts, polyphenoxidase in Beta vulgaris. Plant physiol, 24: 1-15.

Baroowa, B. and Gogoi, N. 2013 Biochemical changes in two Vigna spp. during drought and subsequent recovery. Indian J.Pl. Physiol., 18 : 319-28.

Barr, H. D. and Weatherley, P. E. 1962. A reexamination of the relative turgidity technique for estimating water deficit in leaves. Australian J Bio. Sci., 15: 413-28.

Bates, L. S., Waldren, R. P. and Teare, I. D. 1973. Rapid determination of free proline for water-stress studies, Plant Soil, 39: 205-07.

Bhardwaj, J. and Yadav, S. K. 2012. Comparative study on biochemical parameters and antioxidant enzymes in a drought tolerant and a sensitive variety of horsegram (Macrotyloma uniflorum) under drought stress. American J. Pl. Physiol., 7(1): 17-29.

Borlaug, N. E. 2007. Sixty-two years of fighting hunger: personal recollections. Euphytica, 157: 287-297.

Farooq, M., Wahid, A., Kobayashi, N., Fujita, D., and Basra, S. M. A. 2009. Plant drought stress: effects, mechanisms and management. Agronomy for Sust.Dev., 29: 185-212.

Gokmen, E., and Ceyhan, E. 2018. Effects of drought stress on growth parameters, enzyme activities and proline content in chickpea genotypes. Bangladesh J. Bot., 44(2), 177-83.

Hossaina, M. M., Xueyi, L., Xusheng, Q., Hon-Ming, L. and Zhanga, J. 2014. Differences between soybean genotypes in physiological response to sequential soil drying and rewetting. Crop J., 2(6): 366-80.

Kavi-Kishor, B. B., Sangam, S., Amrutha, R. N., Laxmi, B. S. and Naidu, K. R. 2005. Regulation of proline biosynthesis, degradation, uptake and transport in higher plants: Its implications in plant growth and abiotic stress tolerance. Current Sci., 88: 424-38.

Kumar, R. R., Karajol, K. and Naik, G. R. 2011. Effect of Polyethylene Glycol Induced Water Stress on Physiological and Biochemical Responses in Pigeonpea (Cajanus cajan L. Millsp.). Recent Res. in Sci. and Tech., 3(1): 148-152.

Manivannan, P., Jaleel, C. A., Sankar, B., Kishorekumar, A., Somasundaram, R., Alagu-Lakshmanan, G. M. and Panneerselvam, R. 2007. Growth, biochemical modifications and proline metabolism in Helianthus annuus L. as induced by drought stress. Colloids and Surfaces B: Biointerfaces, 59: 141-149. 
Mattioli, R., Costantino, P. and Trovato, M. 2009. Proline accumulation in plants. Plant Signaling and Behavior, 4(11): 1016-18.

Merah, O. 2001. Potential importance of water status traits for durum wheat improvement under Mediterranean conditions. J. Agri. Res., 137: 13945.

Muscolo, A., Sidari, M., Anastasi, U., Santonoceto, C. and Maggio, A. 2014. Effect of PEG-induced drought stress on seed germination of four lentil genotypes. J. Pl. Interactions, 9(1): 354-63.

Ritchie, S. W., Nguyan, H. T. and Holaday, A. S. 1990. Leaf Water content and gas exchange parameters of two wheat genotypes differing in drought resistance. Crop Sci., 30: 105-11.

Rizvi, A. H., Dwivedi, V. K., Sairam, R. K., Yadav, S. S., Bharadwaj, C., Sarker, A. and Alam, A. 2014. Physiological studies on moisture stress tolerance in chickpea (Cicer arietinum L.) genotypes. Int. J Sci, Res. Agric. Sci., 1(2): 23-31.

Saglam, A., Terzi, R. and Demiralay, M. 2014. Effect of polyethylene glycol induced drought stress on photosynthesis in two chickpea genotypes with different drought tolerance. Acta Biologica Hungarica, 65(2): 178-88.
Sankar, B., Jaleel, C. A., Manivannan, P., Kumar, A.K., Somasundaram, R. and Panneerselvam, R. 2007. Drought induced biochemical modifications and proline metabolism in Abelmoschus esculentus (L.) Moench. Acta Botanica Croatica, 66(1): 4356.

Siddiqui, M. H., Al-Khaishany, M. Y., Al-Qutami, M. A., Al-Whaibi, M. H., Grover, A., Ali, H. A., AlWahibi, M. S. and Bukhari ,N. A. 2015. Response of Different Genotypes of Faba Bean Plant to Drought Stress. Int. J. Mol. Sci, 16(5): 10214227.

Smirnoff, N. 1995. Antioxidant systems and plant response to the environment. In: Smirnoff V (Ed.), Environment and Plant Metabolism: Flexibility and Acclimation, BIOS Scientific Publishers, Oxford, UK.

Talukdar, D. 2013.Comparative morpho-physiological and biochemical responses of lentil and grass pea genotypes under water stress. J. Natural Sci., Biol. Med., 4(2): 396-402.

Vinocur, B. and Altman, A. 2005. Recent advances in engineering plant tolerance to abiotic stress: Achievements and limitations. Curr. Opinion in Biotech., 16: 123-32. 